\title{
CARMA: specifications and status
}

\section{Anthony J. Beasley, Stuart N. Vogel}

Anthony J. Beasley, Stuart N. Vogel, "CARMA: specifications and status," Proc. SPIE 4855, Millimeter and Submillimeter Detectors for Astronomy, (17 February 2003); doi: 10.1117/12.459357

Event: Astronomical Telescopes and Instrumentation, 2002, Waikoloa, Hawai'i, United States 


\title{
CARMA: Specifications \& Status
}

\author{
Anthony J. Beasley ${ }^{\mathrm{a}}$ \& Stuart N. Vogel ${ }^{\mathrm{b}}$

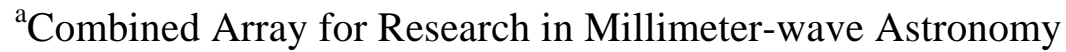 \\ Caltech Owens Valley Radio Observatory \\ PO Box 968 Big Pine, CA 93513 \\ tbeasley@ovro.caltech.edu \\ ${ }^{b}$ Department of Astronomy, University of Maryland \\ College Park, MD 20742 \\ vogel@astro.umd.edu
}

\begin{abstract}
The Combined Array for Research in Millimeter-wave Astronomy (CARMA) is a 23-antenna heterogeneous millimeter array under construction in the White/Inyo Mountains of eastern California. CARMA will merge the existing Owens Valley and Berkeley-Illinois-Maryland Association arrays into a single instrument focusing on pure research, technology development and student training. A new high-altitude site will enable routine $205-265 \mathrm{GHz}$ observing, and may allow observations in the $345 \mathrm{GHz}$ window. Eight additional 3.5-m antennas from the University of Chicago will also be integrated into CARMA when not imaging the Sunyaev-Zel'dovich effect towards clusters of galaxies.

At first light, the array will observe at 12,3 and $1.3 \mathrm{~mm}$ using a mix of SIS and MMIC-based receivers. A new, highly flexible correlator incorporating reprogrammable FPGA technology will process configurable subsets of the antennas specified according to the science objectives. Leading-edge water vapor radiometers will be used to correct for atmospheric opacity and signal phase fluctuations. CARMA will be capable of both high resolution and wide-field imaging, covering a range of angular scales unmatched by any current or planned millimeter-wave instrument. The high sensitivity, sub-arcsecond angular resolution and excellent $u v$-coverage of CARMA will ensure major advances in studies of the universe. The array will provide high-fidelity resolved images of solar-system objects, protostars, protoplanetary disks, and galaxies both nearby and at high redshift - directly addressing many key research areas in astronomy and astrophysics.
\end{abstract}

Keywords: Radio Astronomy; Millimeter Interferometry; Antennas; Correlator; Water Vapor Radiometry

\section{INTRODUCTION}

The CARMA Project is a collaboration of the California Institute of Technology Owens Valley Radio Observatory, the Radio Astronomy Laboratory at the University of California (Berkeley), the Laboratory for Astronomical Imaging at the University of Illinois (Urbana-Champaign), and the Laboratory for Millimeter-wave Astronomy at the University of Maryland. The partner universities and the National Science Foundation have contributed funding for the project. In addition to combining the existing arrays (Fig. 1), the CARMA Project will integrate the University of Chicago Sunyaev-Zel'dovich Array (SZA). The goal of the CARMA Project is to build a flexible leading-edge array dedicated to millimeter astronomy research and student training.

\section{SPECIFICATIONS}

CARMA will be a heterogeneous array, comprised of six 10.4-m and nine 6.1-m telescopes (OVRO+BIMA), and eventually, eight 3.5-m telescopes (SZA). Four configurations of the OVRO and BIMA telescopes (denoted A, B, C, and $\mathrm{D}$ in Table 1), with baselines of $7 \mathrm{~m}$ to $2 \mathrm{~km}$, will yield high fidelity images on angular scales from $30^{\prime \prime}$ to 0.1 " at $1 \mathrm{~mm}$. With the addition of the smaller SZA telescopes (typically in a compact configuration less than $100 \mathrm{~m}$ in diameter) and a single-dish observing capability on the 10.4-m antennas, CARMA will image the universe on all angular scales greater 
than 0.1". Wide-field mosaicing will be standard, and snapshot imaging will become routine. Although CARMA will not initially have dual polarization; full-polarization capability is planned for later development.

\section{Receivers}

Existing receivers and planned upgrades/developments will be integrated into CARMA using a new highly-flexible LO/IF system. At commencement of operations, the frequency coverage available will be:

3-mm band: $84-116 \mathrm{GHz}$ (CARMA \& SZA); 70-84 GHz (9 x 6.1-m antennas only)

1-mm band: $215-270 \mathrm{GHz}$ (CARMA)

Some of the astrophysically-important molecular lines in these bands include $\mathrm{HCN} 1-0(88 \mathrm{GHz}), \mathrm{N}_{2} \mathrm{H}^{+} 1-0(93 \mathrm{GHz})$, CS 2-1 (98 GHz), CO 1-0 (115 GHz), SiO 5-4 (217 GHz), CO 2-1 (230 GHz), CS 5-4 (245 GHz), HCO ${ }^{+} 4-3$ (267 GHz). The expected sensitivity of CARMA in these two bands is indicated in Table 1.

Table 1: CARMA First-light Sensitivity

\begin{tabular}{|c|c|c|c|c|c|c|c|c|c|}
\hline \multirow{2}{*}{\multicolumn{3}{|c|}{$\begin{array}{l}\text { CONTINUUM SENSITIVITY } \\
4 \text { GHZ BW 1-POLARIZATION }\end{array}$}} & \multicolumn{7}{|c|}{$\begin{array}{c}\text { ANGULAR RESOLUTION \& LINE SENSITIVITY } \\
\text { (1 KM/S CHANNEL, 1-POLARIZATION) }\end{array}$} \\
\hline & & & \multicolumn{4}{|c|}{$100 \mathrm{GHZ}$} & \multicolumn{3}{|c|}{$230 \mathrm{GHZ}$} \\
\hline $\begin{array}{l}\text { Freq } \\
\mathrm{GHz}\end{array}$ & $\begin{array}{l}1-\min \\
\mathrm{mJy}\end{array}$ & $\begin{array}{l}5-\mathrm{hr} \\
\mathrm{mJy}\end{array}$ & Config & $\underset{\sim}{\text { Beam }}$ & 1-min & $\begin{array}{c}5-h r \\
K\end{array}$ & $\underset{*}{\text { Beam }}$ & 1-min & $\begin{array}{c}5-h r \\
K\end{array}$ \\
\hline 100 & 1.0 & 0.06 & $\mathrm{D}$ & 6.3 & 0.4 & 0.03 & 2.7 & 1.0 & 0.06 \\
\hline \multirow[t]{3}{*}{230} & 3.3 & 0.19 & C & 2.5 & 2.8 & 0.14 & 1.1 & 6.2 & 0.4 \\
\hline & & & B & 1.0 & 18 & 1.0 & 0.4 & 39 & 2.3 \\
\hline & & & $A$ & 0.4 & 112 & 6.5 & 0.2 & 243 & 14 \\
\hline
\end{tabular}

Assumptions: 6 × $10.4 \mathrm{~m}$ antennas $+9 \times 6.1 \mathrm{~m}$ antennas (i.e. no SZA), $\mathrm{T}_{\mathrm{SYS}} \sim 80 \mathrm{~K}$ at $3 \mathrm{~mm}, 140 \mathrm{~K}$ at $1 \mathrm{~mm} ; 70 \%$ antenna efficiency. Resolution indicated is $/ \mathrm{D}_{\max }$, i.e. no tapering and uniform weighting.

\section{First-light CARMA Correlator}

At CARMA first light, the 15-station correlator will be an extension of the FPGA-based COBRA correlator currently nearing completion at OVRO. The $4 \mathrm{GHz}$ first-light bandwidth will be available as eight $500 \mathrm{MHz}$ bands which can be tuned anywhere within the $4 \mathrm{GHz}$ receiver band. Each of these $500 \mathrm{MHz}$ bands can be "channelized" as indicated in the following table. The total velocity coverage can be increased by combining multiple bands.

Different configurations of the correlator will be appropriate for different science programs. For example, the velocity fields of planetary atmospheres will be traced using the narrowest bandwidths, while the full bandwidth extent of the correlator will permit the first measurements deeper into the atmosphere near to and slightly in excess of 1 bar (substantially deeper than any current array, including OVRO). All $500 \mathrm{MHz}$ bands can be lined up contiguously (i.e. 4 $\mathrm{GHz}$ ) to cope with the strong pressure broadening of the spectral lines. The $4 \mathrm{GHz}$ bandwidth will also make $1 \mathrm{~mm}$ searches for $\mathrm{CO}$ emission from high redshift galaxies much more feasible, even starting from coarse optical redshift information. 
Table 2: Single 500 MHz-band CARMA Correlator Parameters (eight bands available)

\begin{tabular}{||c|c|c|c|c|c||}
\hline $\begin{array}{c}\text { BANDWIDTH } \\
(\mathrm{MHZ})\end{array}$ & CHANNELS & $\begin{array}{c}3 \mathrm{MM} \\
\text { CHANNEL } \\
\text { SPACING } \\
(\mathrm{KM} / \mathrm{S})\end{array}$ & $\begin{array}{c}\text { 3 MM } \\
\text { VELOCITY } \\
\text { WIDTH } \\
(\mathrm{KM} / \mathrm{S})\end{array}$ & $\begin{array}{c}1 \mathrm{MM} \\
\text { CHANNEL } \\
\text { SPACING } \\
(\mathrm{KM} / \mathrm{S})\end{array}$ & $\begin{array}{c}1 \mathrm{MM} \\
\text { VELOCITY } \\
\text { WIDTH } \\
(\mathrm{KM} / \mathrm{S})\end{array}$ \\
\hline 512 & 16 & 96 & 1536 & 32 & 512 \\
\hline 256 & 32 & 24 & 768 & 8 & 128 \\
\hline 128 & 64 & 6 & 384 & 2 & 128 \\
\hline 64 & 128 & 1.5 & 192 & 0.5 & 64 \\
\hline 32 & 128 & 0.75 & 96 & 0.25 & 32 \\
\hline 8 & 128 & 0.18 & 24 & 0.06 & 8 \\
\hline 2 & 128 & 0.05 & 6 & 0.01 & 2 \\
\hline
\end{tabular}

\section{CARMA Capabilities}

CARMA will approach the Very Large Array in its angular resolution, image fidelity, angular dynamic range (ratio of min to max spacing), and mapping speed. As Table 3 illustrates, it will far exceed other $\mathrm{mm}$ and submm arrays (except ALMA) in these capabilities.

Table 3: CARMA Comparison to Existing Arrays

\begin{tabular}{|c|c|c|c|c|c||}
\hline & \multicolumn{2}{|c|}{$\begin{array}{c}\text { BASELINE (M) } \\
\text { MIN }\end{array}$} & $\begin{array}{c}\text { ANGULAR } \\
\text { DYNAMIC } \\
\text { RANGE }\end{array}$ & \# BASELINES & $\begin{array}{c}\text { COLLECTING } \\
\text { AREA (SQ M) }\end{array}$ \\
\hline CARMA & 4 & 2000 & 500 & 253 & 850 \\
\hline OVRO & 18 & 440 & 24 & 15 & 510 \\
\hline BIMA & 7 & 1900 & 270 & 45 & 292 \\
\hline SMA & 8 & 508 & 64 & 28 & 226 \\
\hline PdB & 24 & 400 & 17 & 15 & 1060 \\
\hline VLA & 40 & 40000 & 1000 & 351 & 13254 \\
\hline
\end{tabular}

CARMA, which will concentrate on millimeter wavelengths, will nicely complement the SMA, which will focus on submillimeter observations. CARMA's $4 \mathrm{GHz}$ bandwidth and high altitude location will provide a significant increase in sensitivity at $1 \mathrm{~mm}$ over the existing OVRO and BIMA arrays. Compared to BIMA, the $1 \mathrm{~mm}$ sensitivity of CARMA will be better by a factor of 18 for continuum and 8 for spectral line work. Compared to OVRO, the improvement will be a factor of 7 for continuum and 5 for spectral line. Significant additional improvements in effective sensitivity can be expected for many observations due to reduced decorrelation resulting from self-calibration and/or WVR radiometry. In 
addition, improved sensitivity and routine observing in the $1 \mathrm{~mm}$ band at the new site will effectively make more of the sky accessible to CARMA. At present, it is difficult to study sources such as Ophiuchus, which transit in the summer months when the weather precludes observing.

The maximum angular resolution achievable with BIMA and CARMA are comparable and a factor of nearly 5 better than OVRO. However, CARMA will provide many more long baselines With the SZA, the number of baselines will increase by a factor of 6 for BIMA and 17 for OVRO; the angular dynamic range will increase by factors of 2 and 20 , respectively. With 253 instantaneous baselines and a decrease in nearly two orders of magnitude in the time required to reach a given sensitivity, CARMA will be able to accomplish in four hours what would have previously required 2 months of daily 8-hr tracks.

Improved Imaging Fidelity

CARMA with its inhomogeneous array of antennas will have unique capability to image at both high angular resolution and in large fields. A simulation demonstrating the improved imaging capability of CARMA is shown in Fig. 2. A single pointing interferometric observation with an array of antennas of one size is insensitive to map structures larger than about one half the primary beam angle of the antennas. While a mosaic of pointings can cover a larger field, it still mostly misses the smooth extended structures in this larger field due to the lack of visibility data in the central hole of the $u v$ plane, corresponding to the minimum antenna separation of one antenna diameter. In principle, a total power map made with one of the array antennas can supply the missing visibility data but typical pointing errors produce large visibility errors in the central region that lead to poor dynamic range in the final maps. If the antenna used for the total power maps has two or three times greater diameter than the array antennas, pointing errors are much less important, and the combined maps have much greater accuracy.

With multiple antenna sizes, the CARMA array can produce accurate large field images. For CARMA, single dish visibilities will derive from maps made with the 10.4-m antennas. The combination of these with interferometer maps made with the $3.5-\mathrm{m}, 6.1-\mathrm{m}$, and $10.4-\mathrm{m}$ antennas observing in mosaic mode will produce accurate overall visibilities and maps with high dynamic range. (The most important feature is the combination of 10.4-m single dish with the 3.5-m array). No other existing array has this capability. Only if the ALMA array of 12-m antennas is supplemented by the auxiliary array of 7-m diameter antennas will it be competitive with CARMA for large field imaging.

A conceptual layout for the CARMA site is shown in Fig. 4. The central array area will contain the control and maintenance buildings required for array support, and the infrastructure to support the compact configurations.

\section{A New High-Altitude Site}

At millimeter wavelengths, water vapor in the first 6,000 to 9,000 feet above sea-level absorbs and distorts the radio signals from space. By placing CARMA at a new high-altitude site, absorption and distortion of the astronomical signals by atmospheric water vapor will be greatly reduced, allowing clearer and more sensitive observations. A number of potential sites in the White/Inyo Mountains of eastern California have analyzed, including Juniper Flat (7900') and Cedar Flat East (7300') (Fig. 3). The improvement is atmospheric opacity compared to either existing 4000' site is typically a factor of two in most metrics - excellent opacity statistics measured at the Juniper Flat site during winter 2002 are presented in Fig. 5.

\section{Transporter \& Relocation}

To move the CARMA antennas between the four configurations a new transporter capable of raising and moving both antenna types is required. We have explored a fifth-wheel trailer design involving a lifting frame connected to a commercial mining/towing vehicle (Fig. 6). In addition to antenna reconfiguration, this transporter will be used to relocate the OVRO (and possibly BIMA) antennas to the new high-altitude site from the valley floor. Options for the transport of the BIMA antennas from the Hat Creek site to the Owens Valley are under investigation; one plan is indicated in Fig. 7. 


\section{Water Vapor Radiometry}

Routine and reliable imaging at $0.1 "$ spatial resolution will be a major capability and science requirement for the CARMA array. In order to exploit the full potential of the longest baselines, the array must be fitted with the radio equivalent of active optics: a system that measures and corrects phase shifts from fluctuating amounts of tropospheric water vapor. Patches of water vapor that drift across the array cause the fringe pattern to shift on the sky, decreasing its point-source sensitivity and smearing flux within a map to reduce its dynamic range. Considerable research is still required to enable accurate calibration of arrays like CARMA and ALMA using these phase correction techniques. BIMA and OVRO will jointly develop and fabricate a common water vapor radiometer (WVR) system and integrate it into the data pipeline coming from the array.

\section{CARMA SCIENCE}

With much more powerful capabilities than existing arrays, CARMA will enable major advances in extragalactic astronomy, from observations of the formation of the first generation of galaxies at $\mathrm{z}>2$ to the conditions and dynamics in nearby galactic bars, nuclear starbursts, and AGN. High resolution CARMA images, combined with complementary data from far infrared (SIRTF, SOFIA) and large optical telescopes (NGST, Keck, and Gemini), will revolutionize our understanding of star formation and galactic evolution both at early epochs and at present. Many important northern declination sources will be accessible to CARMA but not to ALMA due to the latter's very southern location.

CARMA will provide key insights into how the first stars and galaxies formed and evolved.

CARMA will detect both dust and lines from these distant sources. $\mathrm{CO}$ has now been detected in numerous high redshift systems, but the sample to date provides few constraints on early cosmic evolution since limited sensitivity biases the current arrays. CARMA's increased sensitivity will extend the sample to many sources and find their luminosity function. As already noted, CARMA's broad bandwidth will make $1 \mathrm{~mm}$ searches for redshifted $\mathrm{CO}$ emission feasible, even starting from coarse optical redshift information. Since the CO 2-1 fluxes are 49 times brighter than CO 1-0, the line intensity becomes brighter faster than the system temperature increases. The $1 \mathrm{~mm}$ band is also the first window for detecting $\mathrm{CO}$ at $z=0.4-0.9$, a redshift range in which optical studies have revealed considerable galactic evolution. With the ability to detect and resolve galaxies at all redshifts, CARMA will revolutionize understanding of the evolution of the molecular gas and dust content of galaxies.

CARMA will be able to determine the density structure of the dark matter in the centers of an extended sample of dwarf galaxies by measuring the two dimensional velocity structure of the $\mathrm{CO}$ emission.

The current disagreement between CDM simulations and observations of the dark matter density structure points to a crisis in our theoretical understanding of the dark matter, or a fundamental problem with the simulations, or flaws in the interpretation of the observations. As a result of CARMA's good sensitivity at high resolution, two-dimensional CO rotation curves will be obtained for a sizable sample of dwarf galaxies, extending the small sample of dark-matter dominated dwarfs observable with the current arrays. This larger sample will make it possible to address the disagreement between theory and observation and will provide us with a deeper understanding of the dynamics of gas and star formation in shallow potentials.

CARMA surveys of GMCs in many galaxies will trace their formation and destruction and map radial gas flows in bars and galactic nuclei.

Surveys such as MAIN and BIMA SONG have shown that key structures exist on a wide range of size scales in galactic disks and nuclei. CARMA's unique combination of heterogeneous imaging, high sensitivity, and high angular resolution, will yield major advances in understanding the molecular ISM in galaxies. With a single 5-hour track at CARMA, all the GMCs with masses greater than $10^{4} \mathrm{M}$ can be detected in a 1-kpc region in a galaxy at a distance of $4 \mathrm{Mpc}$. For comparison, in a similar area in the Milky Way near the Sun there are 20 GMCs with masses greater than $10^{4} \mathrm{M}$. With a linear resolution of less than $20 \mathrm{pc}$ at distances less than $4 \mathrm{Mpc}$ (comparable to the effective linear resolution of some Milky Way surveys), CARMA can undertake unbiased surveys of GMCs in a range of environments to determine the 
role of kinematics, gas surface density, metallicity, pressure, radiation field, density waves, and other factors on the formation and destruction of GMCs, and on the rate at which GMCs form massive stars.

CARMA Sunyaev-Zel'dovich Effect imaging of the high redshift universe will measure the evolution of largescale structure and determine critical cosmological parameters.

Using the eight 3.5-m telescopes, CARMA will survey 12 square degrees for the Sunyaev-Zel'dovich Effect (SZE) from distant galaxy clusters to yield a complete inventory of clusters above a mass limit for all redshifts extending to the epoch of cluster formation. This sample will set tight constraints on the matter and energy densities of the universe as well as the Hubble constant and the acceleration of the universe. Detailed follow-up full CARMA imaging of the clusters found in the survey will be used to determine their gas properties and evolution. Such imaging is critical, for example, before the equation of state of the dark energy can be extracted from the yields of the proposed larger, but lower resolution, cluster surveys.

High-resolution CARMA mosaics of star-forming complexes will survey cluster-forming cores for protostellar condensations with masses down to 0.03 solar masses to shed light on the origins of the stellar initial mass function (IMF). Similar surveys of young clusters will help establish evolutionary time scales for planet formation.

Star formation and the interstellar medium (ISM) encompass structures ranging from individual protostellar cores with sub-arcsecond size-scales to star-forming complexes many arcminutes in extent. This broad range of fundamental size scales is well matched to CARMA's unique wide-field and high resolution imaging capabilities. With CARMA, large mosaics of molecular line and continuum emission will be attainable approximately ten times faster, and with higher dynamic range, than is currently possible. A sensitivity of $\sim 1 \mathrm{mJy} / \mathrm{beam}$ at $1.3 \mathrm{~mm}$ over $5^{\prime}$ x $5^{\prime}$ can be reached in only 1 transit, allowing protostellar condensations with masses down to those of brown dwarfs to be detected.

CARMA will probe the evolution of structure, kinematics, and chemistry in protostellar cores and protoplanetary disks on linear scales of $17 \mathrm{AU}$ in the nearest star-forming clouds.

CARMA will provide the most sensitive view yet of the kinematics, structure, and chemistry of this early stellar environment and contribute to a broad understanding of how stars and planets form and evolve. It will be possible to test emerging chemical models and clarify the origins of the primitive solar system. An important new project for CARMA will be the exploration of pre-biotic chemistry in low mass star-forming cores similar to the proto-solar nebula. When combined with emerging infrared capabilities (the proposed NASA AstroBiology Explorer mission will obtain spectroscopic observations in the 2.5 to $16 \mathrm{~m}$ range), the sensitivity and imaging performance of CARMA will open up a new era for the detection and identification of the new molecules important to astrobiology.

CARMA will obtain measurements of the magnetic field strength and structure of dense, star-forming cores.

Polarization measurements with arrays are very sensitivity limited, and the order of magnitude improvement that CARMA brings will lead to an explosion of new results in this area. It will be possible to map magnetic field strength and morphology with a full order of magnitude higher angular resolution than other (single-antenna) instruments. For the first time, Zeeman mapping of tracers of dense gas will be possible. Together with dust and spectral line linear polarization maps, this will enable us to infer the full three dimensional magnetic field. It should be possible to address fundamental questions about the role of magnetic fields in star formation through an understanding of the relative importance of regular fields and turbulence for cloud support, of the mechanism for loss of angular momentum in collapsing cores, and of the generation of protostellar bipolar outflows.

CARMA's sensitivity will at last enable size determinations of the Kuiper belt objects (KBO's), while its superb mosaicing capabilities will enable novel studies of planetary surfaces and atmospheres and, especially, comets.

Many of the important observational targets in the solar system display changes in their emission properties on time scales similar to or shorter than the Earth's rotation period. Detailed studies of the Sun, the atmospheres of terrestrial 
and Jovian planets, and the comae of comets will thus exploit CARMA's capability for rapid imaging over a large range of spatial scales.

Comets are among the most primitive bodies in the solar system and may still preserve the mixture of interstellar and nebular material prevailing at their origin sites in the outer solar nebula. The combination of high angular resolution, large field of view, and snapshot imaging compensate for the uncertain ephemeredes of new comets, their rapid variability and the spatial offset of the molecular line peaks relative to the nuclei. Since, with 15 elements and a continuum sensitivity of $<25 \mathrm{mJy}$ in 1 second, useful images can be attained in only a few minutes, CARMA will greatly enlarge the sample of available targets. Imaging of comets with production rates at least 100 times lower will be feasible, and the dramatically improved sensitivity will allow, in a single track, the detection of any cometary nucleus greater than $2 \mathrm{~km}$ in size and within $1 \mathrm{AU}$ of the earth.

\section{STATUS}

In 2001 an application for the new site was submitted to the US Forest Service, and in 2002 federal and state environmental review of the site candidates was initiated. A final decision on the new site is expected early in 2003. A geographically-distributed engineering design and analysis process has been developed, and preliminary and critical design reviews of major CARMA subsystems are underway. Correlator production is scheduled to commence in early 2003. Our current plan is to move the OVRO antennas to the new site first during 2004; at the same time, the BIMA array will be relocated to the Owens Valley (valley floor or high site). Reconstruction and integration of all BIMA antennas and transition to early CARMA operations are expected in 2005.

\section{ACKNOWLEDGEMENTS}

This paper has been produced on behalf of the CARMA consortium, and represents the work of scientists, engineers and technicians at the University of California (Berkeley), the University of Maryland (College Park), the University of Illinois (Urbana-Champaign) and the California Institute of Technology Owens Valley Radio Observatory. The OVRO and BIMA arrays and CARMA are partially supported by National Science Foundation grants NSF-9981546, NSFAST9983108, NSF-AST9981363, NSF-AST991289 and NSF 011616558. 

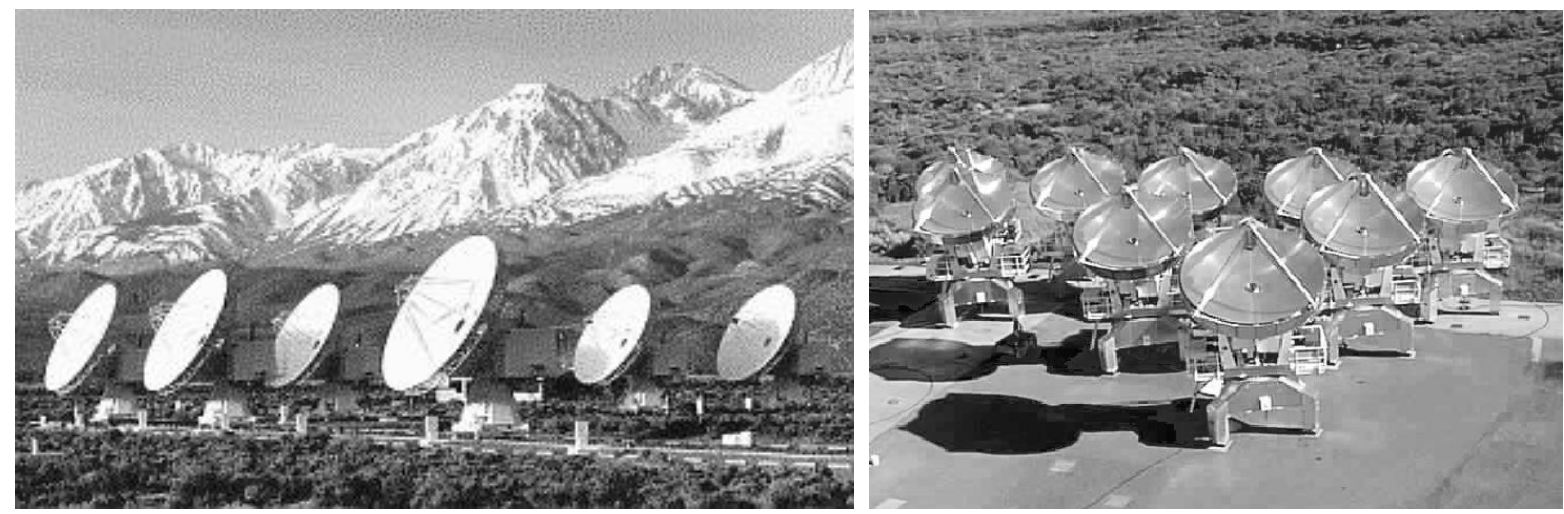

Figure 1: Owens Valley Radio Observatory six 10.4-m antennas (left); Berkeley-IllinoisMaryland Association nine 6.1-m antennas (right)

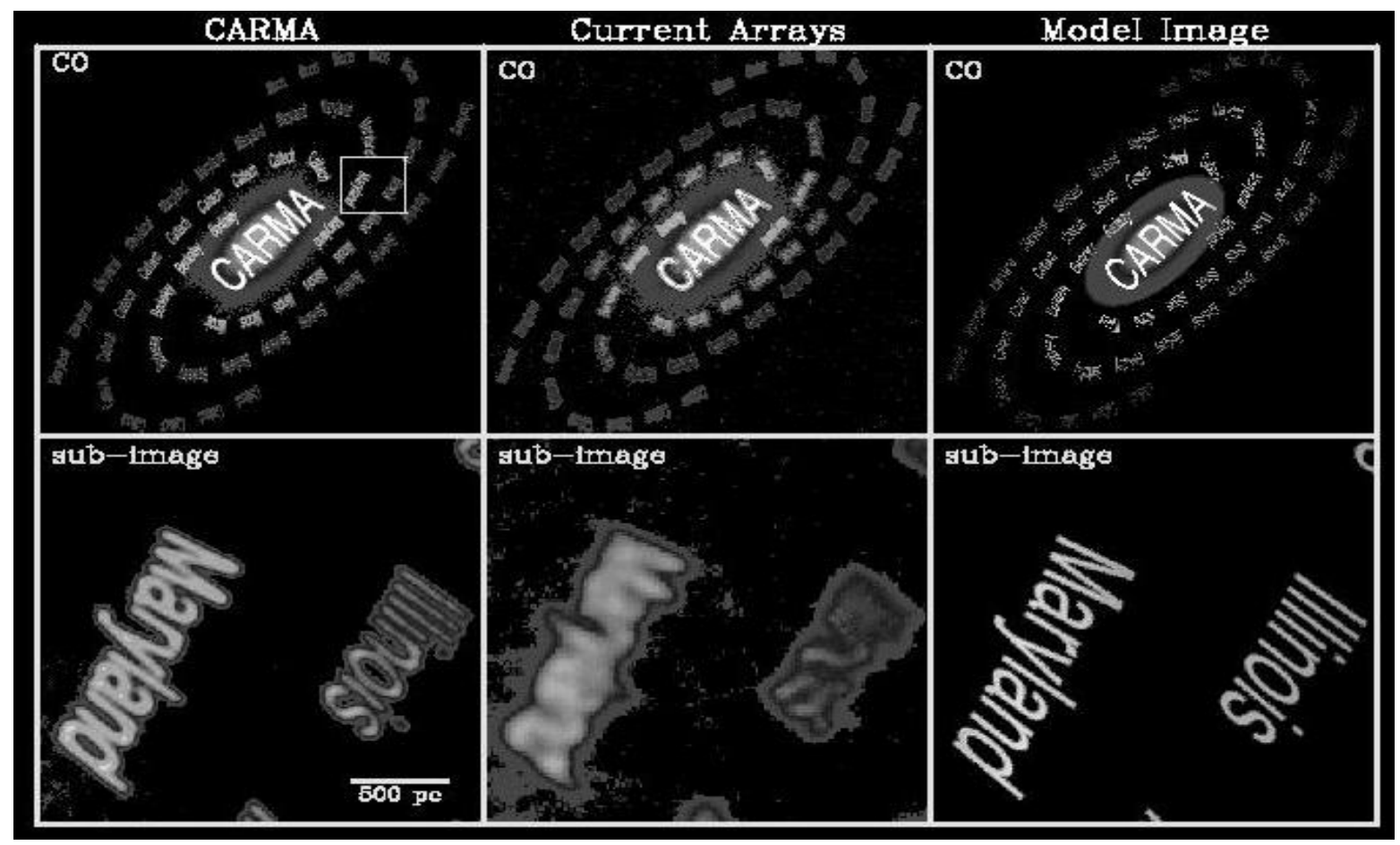

Figure 2: Simulation of CARMA CO imaging capability, using text to represent molecular clouds for a simulated spiral galaxy in the Virgo Cluster. CARMA more accurately reproduces both large and small-scale structure. The small box indicated in the top left image is expanded along the bottom row. 

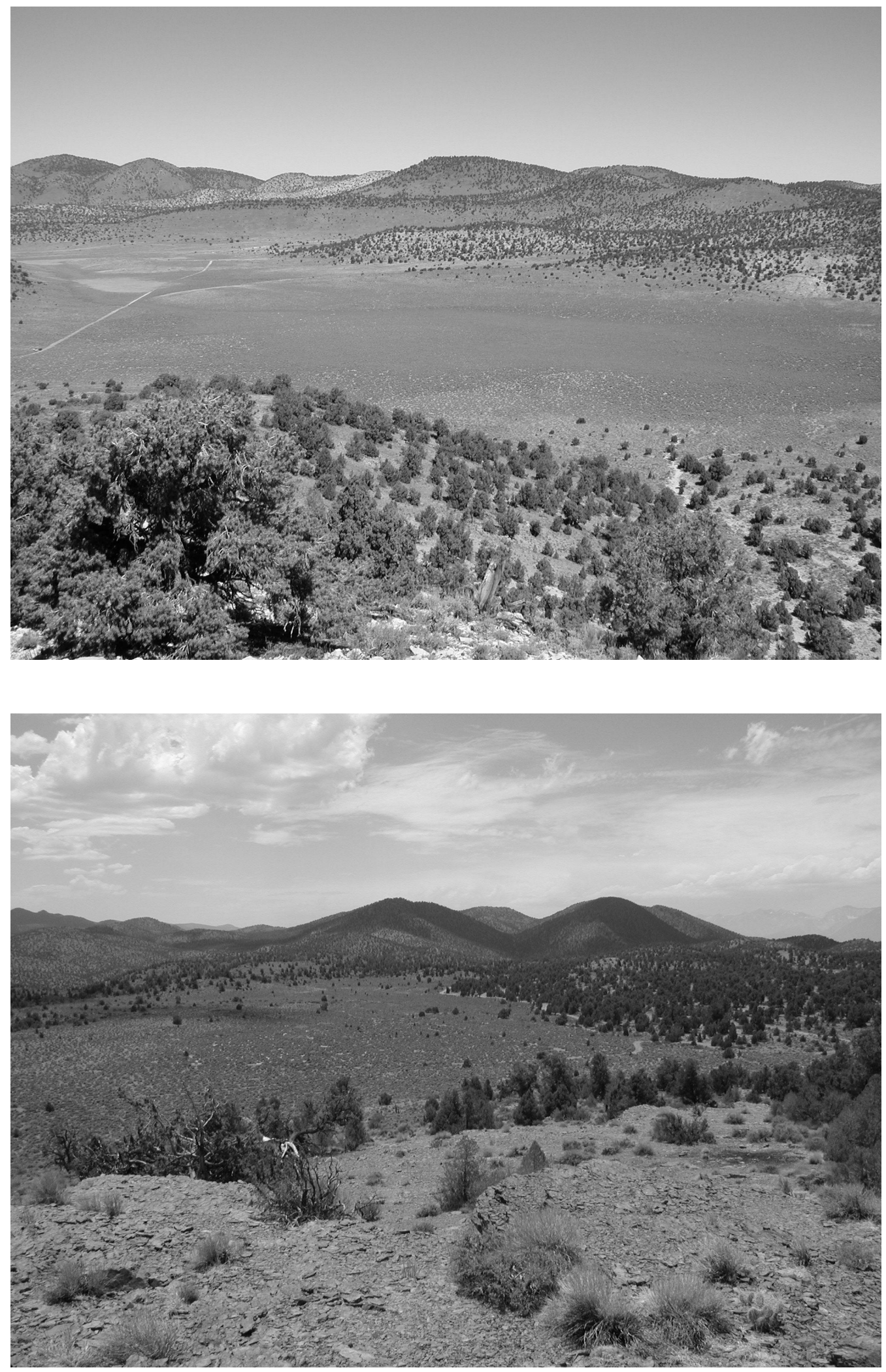

Figure 3: CARMA candidate sites - Juniper Flat (above), Cedar Flat East (below). 


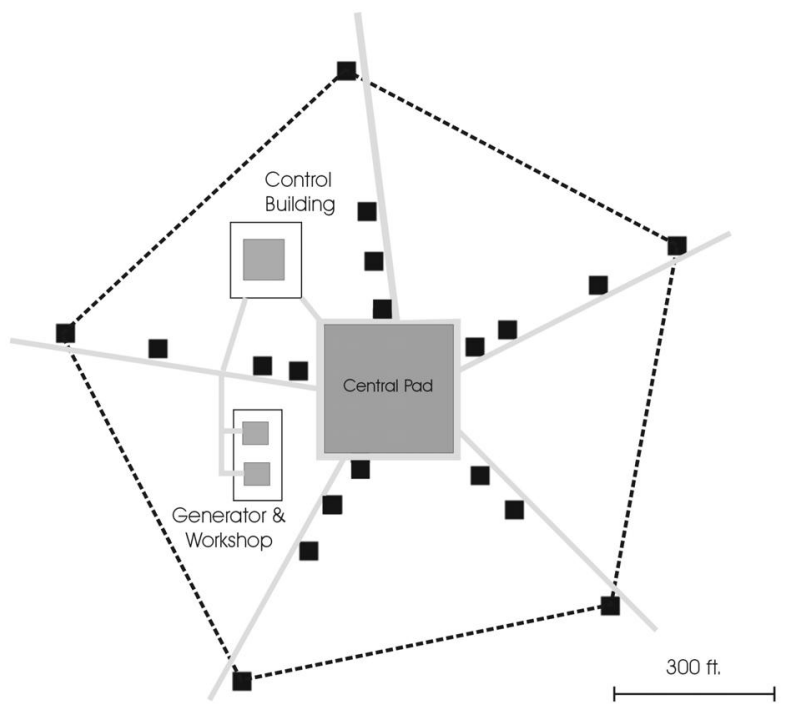

Legend

- Antenna Pad

-.-- Central Array Area

Connector Road

Building \& Grounds

Figure 4: CARMA Central Array Area

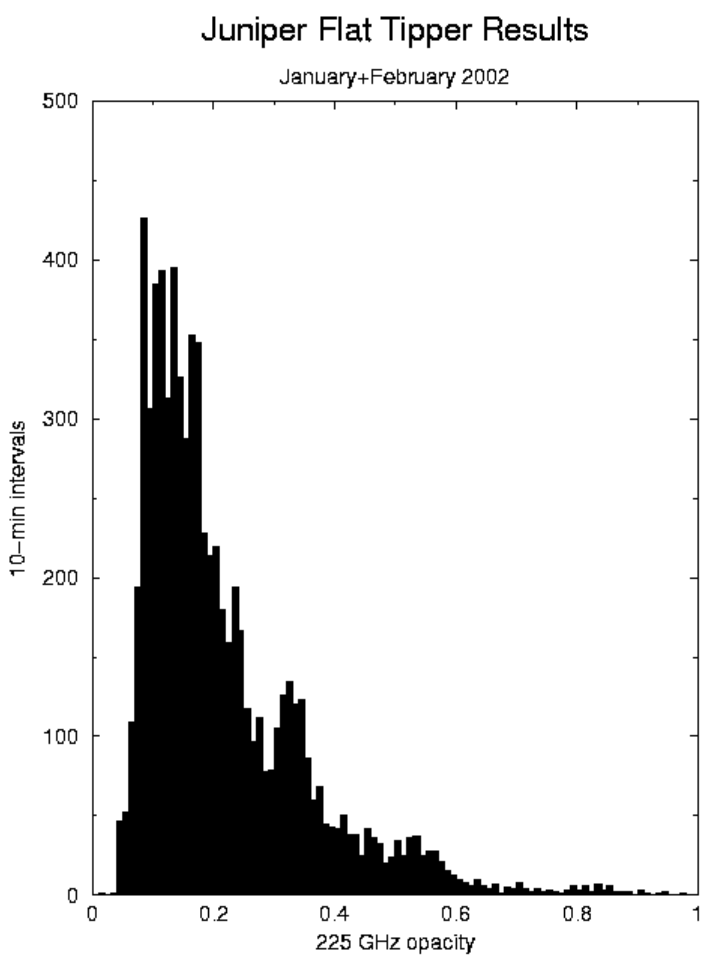

Figure 5: Juniper Flat Atmospheric Monitoring results - Winter 2002 


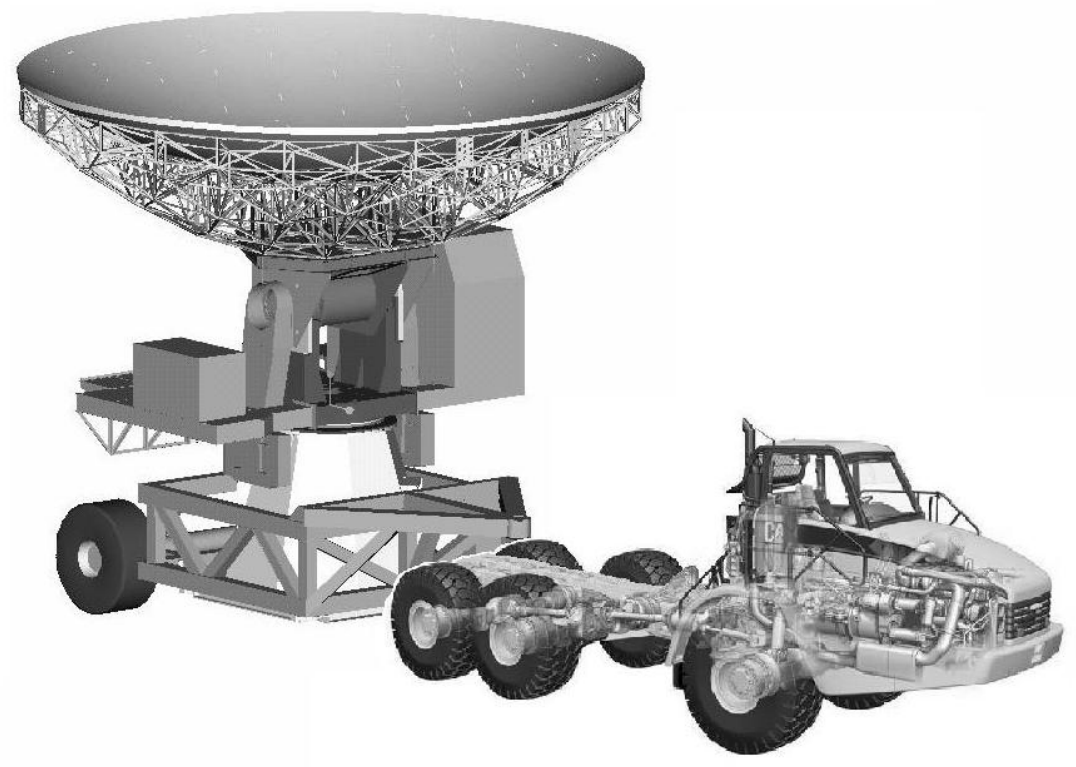

Figure 6: CARMA Antenna Transporter concept

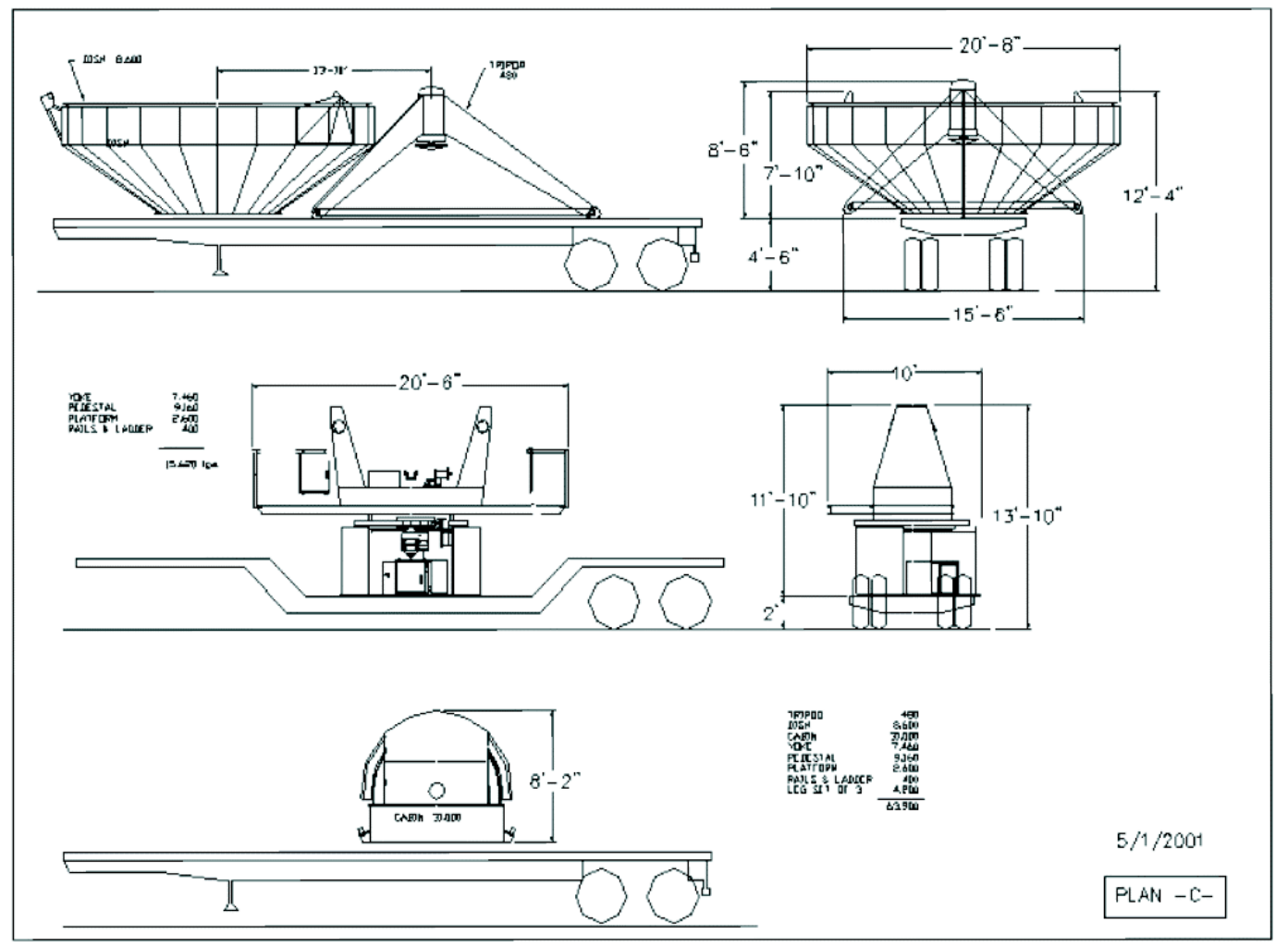

Figure 7: One transport plan for the BIMA antennas. In this plan, each antenna is moved using three trucks. 